\title{
Growth Plate, Bone and Calcium
}

\author{
Terhi Heino ${ }^{a}$, Dov Tiosanob, and Lars Sävendahk \\ arthopaedic Research Unit, University of Turku, Finland \\ bediatric Endocrinology, Rambam Medical Center, Haifa, Israel \\ cPediatric Endocrinology Unit, Department of Woman and Child Health, Karolinska Institutet, Stockholm, \\ Sweden
}

\begin{abstract}
Studies of bone biology have been complicated by the highly organized structures and complex cellular biology. Thanks to new models and technologies, our view and understanding of the biological importance of the skeleton has expanded dramatically in the last years. We first take the opportunity to highlight a few papers discussed in this chapter. The possibility of using C-type natriuretic peptide as a novel therapeutic strategy for skeletal dysplasias gives new hope for the treatment of achondroplasia. Two papers demonstrating that oxytocin is a bone-anabolic hormone represent a new paradigm. The recent discovery of the extracellular calcium-sensing receptor as a critical modulator of skeletal development was chosen as the mechanism of the year. Other new mechanisms include the demonstration of prolactin as a regulator of bone metabolism and the discovery of a new estrogen receptor involved in the regulation of bone growth. An important observation for clinical practice is the finding that late menarcheal age can be considered as a risk factor for osteoporosis. We also included a preclinical study indicating that osteocytes are target cells to increase bone mass, a finding which may lead to new treatment options in patients with osteoporosis. Clinical trials are represented by 2 papers that potentially could open up a new therapy of hypoparathyroidism in children and another paper demonstrating that the effectiveness of vitamin $D$ supplementation is independent of the dosing regimen. A concept revised is represented by a paper showing a new player involved in hypophosphatemic rickets. New anti-cancer and anti-inflammatory drugs are reported to interfere with bone growth representing new concerns. Giving some food for thought is a paper suggesting that aspirin might have the capacity to prevent osteoporosis. Finally we chose two reviews: one nicely summarizing the experiences of the use of bisphosphonates in the treatment of osteogenesis imperfecta, and another proposing a new way of classifying rickets based on the etiology of the underlying hypophosphatemia.
\end{abstract}

\section{New hope: one step further towards an effective treatment of achondroplasia}

\section{Systemic administration of C-type natriuretic peptide as a novel therapeutic strategy for skeletal dysplasias}

Yasoda A, Kitamura H, Fujii T, Kondo E, Murao N, Miura M, Kanamoto N, Komatsu Y, Arai H, Nakao K

Department of Medicine and Clinical Science, Kyoto University Graduate School of Medicine, Kyoto, Fuji-Gotemba

Research Laboratories, Chugai Pharmaceutical Co., Ltd., Shizuoka, Japan

yasoda@kuhp.kyoto-u.ac.jp and nakao@kuhp.kyoto-u.ac.jp

Endocrinology 2009; DOI: 10.1210/en.2008-1676

Background: C-type natriuretic peptide (CNP), a member of the natriuretic peptide family, is a potent stimulator of endochondral bone growth. Targeted overexpression of a CNP transgene in the growth plate has been shown to rescue the impaired bone growth observed in mouse model of achondroplasia (Ach), the most frequent form of human skeletal dysplasias.

Methods: To elucidate whether or not the systemic administration of CNP is a novel drug therapy for skeletal dysplasias, the authors investigated the effects of plasma CNP on impaired bone growth in Ach mice that specifically overexpress CNP in the liver under the control of human serum amyloid P component promoter, or in those treated with a continuous CNP infusion system. 
Results: The data show that increased plasma CNP from the liver or by intravenous administration of synthetic CNP-22 rescued the impaired bone growth phenotype of Ach mice without significant adverse effects.

Conclusion: Treatment with systemic CNP could be a potential therapeutic strategy for skeletal dysplasias, including achondroplasia in humans.

Achondroplasia is caused by constitutive active mutation of FGF receptor 3 (FGFR3), which results in disturbed proliferation and differentiation of growth plate chondrocytes followed by impaired endochondral bone growth [1]. Growth hormone has been shown to have only a minimal effect in patients with achondroplasia [2] and today there is no effective treatment available. As discussed in Yearbook 2008, C-type natriuretic peptide (CNP) is a potent stimulator of endochondral bone growth [3]. Mice with targeted overexpression of CNP in cartilage exhibit prominent skeletal overgrowth [4] while mice depleted with CNP are dwarf due to impaired endochondral bone growth [5]. The authors of this paper have previously demonstrated that cartilage-specific overexpression of a CNP transgene rescues the impaired endochondral bone growth in a mouse model of achondroplasia with targeted expression of constitutive active FGFR3 in cartilage (Ach mice), by restoring the decreased matrix production in Ach growth plates through inhibition of FGFR3-mediated MAPK signaling pathway [4]. In this paper, the same authors investigated the effects of plasma CNP on impaired bone growth in Ach mice that specifically overexpress CNP in the liver and in those treated with a continuous CNP-22 infusion system. The data convincingly show that increased plasma CNP from the liver or by intravenous administration of synthetic CNP-22 rescued the impaired bone growth phenotype of Ach mice without significant adverse effects. Nevertheless, safety issues with CNP need further study, as only short-term potential toxicity has been examined in the current study. It is also important to point out that continuous subcutaneous infusion of similar doses of CNP-22 was ineffective which may be due to subcutaneous degradation of CNP-22. As CNP could successfully stimulate the endochondral bone growth of wild-type mice; it could potentially be used for skeletal dysplasias other than achondroplasia. However, it is important to point out that the animals were treated as of their weaning age of 3 weeks and we therefore do not yet know whether this is a requirement for successful therapy. Although further research is needed, systemic administration of CNP or CNP analogs provides a novel promising therapeutic strategy for human skeletal dysplasias, including achondroplasia.

\section{New paradigms: oxytocin is a bone-anabolic hormone}

\section{Oxytocin controls differentiation of human mesenchymal stem cells and reverses osteoporosis}

Elabd C, Basillais A, Beaupied H, Breuil V, Wagner N, Scheideler M, Zaragosi LE, Massiera F, Lemichez E, Trajanoski Z, Carle G, Euller-Ziegler L, Ailhaud G, Benhamou CL, Dani C, Amri EZ

ISBDC, Université de Nice Sophia-Antipolis, CNRS, Nice, France

amri@unice.fr

Stem Cells 2008;26:2399-2407

Background: Osteoporotic bone loss constitutes a major worldwide public health burden characterized by enhanced skeletal fragility. Whereas an increase in bone resorption is considered as the main contributor to bone loss, the process is also accompanied by increased bone marrow adiposity. Osteoblasts and adipocytes share the same precursor cell and an inverse relationship exists between the two lineages. To develop new therapeutic treatments, it is important to identify signaling pathways that stimulate the osteogenesis of mesenchymal stem cells (MSCs) at the expense of adipogenesis.

Methods: The authors identified the oxytocin receptor pathway as a potential regulator of the osteoblast/ adipocyte balance of human multipotent adipose-derived stem cells by transcriptomic analysis. The effects of oxytocin on the osteoblast/adipocyte balance in vitro and the potential of oxytocin to prevent ovariectomy (OVX)-induced bone loss in vivo were tested. In addition, levels of circulating oxytocin were measured in OVX rats and postmenopausal women. 
Results: Both oxytocin and carbetocin (a stable oxytocin analog) negatively modulated adipogenesis while promoting osteogenesis in both human multipotent adipose-derived stem cells and human bone marrow MSCs. Consistent with these observations, OVX mice and rats, which become osteoporotic and exhibit disequilibrium of this balance, had significantly decreased oxytocin levels compared to sham-operated controls. Subcutaneous oxytocin injection reversed bone loss and reduced marrow adiposity in OVX mice. Clinically, plasma oxytocin levels were significantly lower in postmenopausal women developing osteoporosis than in their healthy counterparts.

Conclusion: These results suggest that plasma oxytocin levels represent a novel diagnostic marker for osteoporosis and that oxytocin administration could be a potential therapy to prevent bone loss.

\section{Oxytocin is an anabolic bone hormone}

Tamma R, Colaianni G, Zhu LL, DiBenedetto A, Greco G, Montemurro G, Patano N, Strippoli M, Vergari R, Mancini L, Colucci S, Grano M, Faccio R, Liu X, Li J, Usmani S, Bachar M, Bab I, Nishimori K, Young LJ, Buettner C, Iqbal J, Sun L, Zaidi M, Zallone A

Department of Human Anatomy and Histology, University of Bari, Bari, Italy

mone.zaidi@mssm.edu or a.zallone@anatomia.uniba.it

Proc Natl Acad Sci USA 2009;106:7149-7154

Background: Oxytocin, a primitive neurohypophyseal hormone, has so far been thought to only modulate lactation and social bonding. Here, the authors attempted to determine whether oxytocin directly affects bone remodeling.

Methods: The skeletal phenotype of oxytocin and oxytocin receptor knockout mice was analyzed by histomorphometry and $\mu \mathrm{CT}$. The effects of local and peripheral injections of oxytocin on bone parameters were studied. Furthermore, in vitro cultures of murine and human osteoblasts and osteoclasts were performed.

Results: The authors demonstrated that oxytocin is a direct regulator of bone mass. Deletion of oxytocin or the oxytocin receptor in male or female mice caused osteoporosis, which resulted from reduced bone formation. Consistent with low bone formation, oxytocin was shown to stimulate the differentiation of osteoblasts to a mineralizing phenotype. The molecular mechanism involved the upregulation of BMP2, which in turn controlled Schnurri-2 and 3, Osterix, and ATF-4 expression. In contrast, oxytocin had dual effects on the osteoclast. It stimulated osteoclast formation both directly and indirectly. On the other hand, oxytocin inhibited bone resorption by mature osteoclasts by triggering cytosolic calcium release and nitric oxide synthesis.

Conclusion: The complementary genetic and pharmacologic approaches presented in this paper revealed oxytocin as a novel anabolic regulator of bone mass, with potential implications for osteoporosis therapy.

The mammalian peptide hormone oxytocin is most well known for its roles in bonding, attachment and female reproduction: it is released after distension of the cervix and vagina during labor and after stimulation of the nipples, thus respectively facilitating birth and breastfeeding. These two papers describe a new potential role for oxytocin as an anabolic regulator of bone mass. Elabd et al. were looking for factors that are differentially regulated during the adipogenic and osteogenic differentiation of mesenchymal stem cells (MSCs) and found the oxytocin receptor gene as a potential candidate. They showed that oxytocin modulates the osteoblast/adipocyte balance of MSCs in vitro and prevents ovariectomy (OVX)-induced bone loss in mice in vivo. In addition, serum and plasma oxytocin levels were decreased both in OVX rats and in postmenopausal women developing bone loss. An important question raised by their findings was whether the effect of oxytocin on the skeleton is direct or central? This was recently addressed in the paper by Tamma et al. who, in knockout mouse models, showed that peripheral oxytocin has a direct and dominant action on the skeleton. The effect was mainly mediated through the stimulation of osteoblast formation, with variable effects on osteoclasts. These data indeed lead to the speculation that oxytocin could potentially be used as a new bone-anabolic therapy. Nevertheless, further experimental studies are needed to verify whether this concept is feasible. Like oxytocin, parathyroid hormone-related protein (PTHrP), another peptide affecting the breast, displays both pro-resorptive and anabolic actions. PTHrP secreted from the breast causes, at least in part, the bone loss that accompanies pregnancy and lactation. Another primitive neuropeptide, calcitonin, which is also increased during pregnancy and lactation, is likely to counteract the stimulatory effects of oxytocin. 


\section{Evidence for direct effects of prolactin on human osteoblasts: inhibition of cell growth and mineralization}

Seriwatanachai D, Krishnamra N, van Leeuwen JP

Faculty of Science, Department of Physiology, Mahidol University, Bangkok, Thailand

j.vanleeuwen@erasmusmc.nl

J Cell Biochem 2009; DOI: 10.1002/jcb.22161

Background: Hyperprolactinemia is one of the risk factors decreasing bone mass which has been believed to be mediated by hypogonadism. However, the demonstration of prolactin receptors in a human osteosarcoma cell line and primary bone cell cultures from mouse calvariae supported the hypothesis of a direct prolactin (PRL) action on bone cells. The aim of this study was to investigate the role of PRL and its signal transduction pathway in the regulation of bone metabolism via osteoblast differentiation.

Methods: Human pre-osteoblasts (SV-HFO) that differentiate in a 3-week period from proliferating preosteoblasts (days 2-7) to extracellular matrix-producing cells (days 7-14), which are eventually mineralized (days 14-21), were used. The concentration of PRL mimicking a lactating period $(100 \mathrm{ng} / \mathrm{ml})$ was used to incubate SV-HFO for 21 days in estrogenic medium. The human PRL receptor mRNA and protein are expressed in SV-HFO.

Results: PRL significantly decreased osteoblast number (DNA content) which was due to a decrease in proliferation. PRL increased osteogenic markers, RUNX2 and ALP, in the early stage of osteoblast differentiation while decreasing it later, suggesting a bidirectional effect. Calcium measurement and Alizarin red staining showed a reduction in mineralization by PRL while having no effect on osteoblast activity or RANKL/OPG mRNA ratio. PRL action on mineralization was not via the PI-3 kinase pathway.

Conclusions: The present study provides evidence of a direct effect of PRL on osteoblast differentiation and in vitro mineralization.

Bone loss occurring during lactation is due to accelerated bone resorption. The trigger for bone resorption is the combination of increased circulating levels of PTHrP, oxytocin from the previous

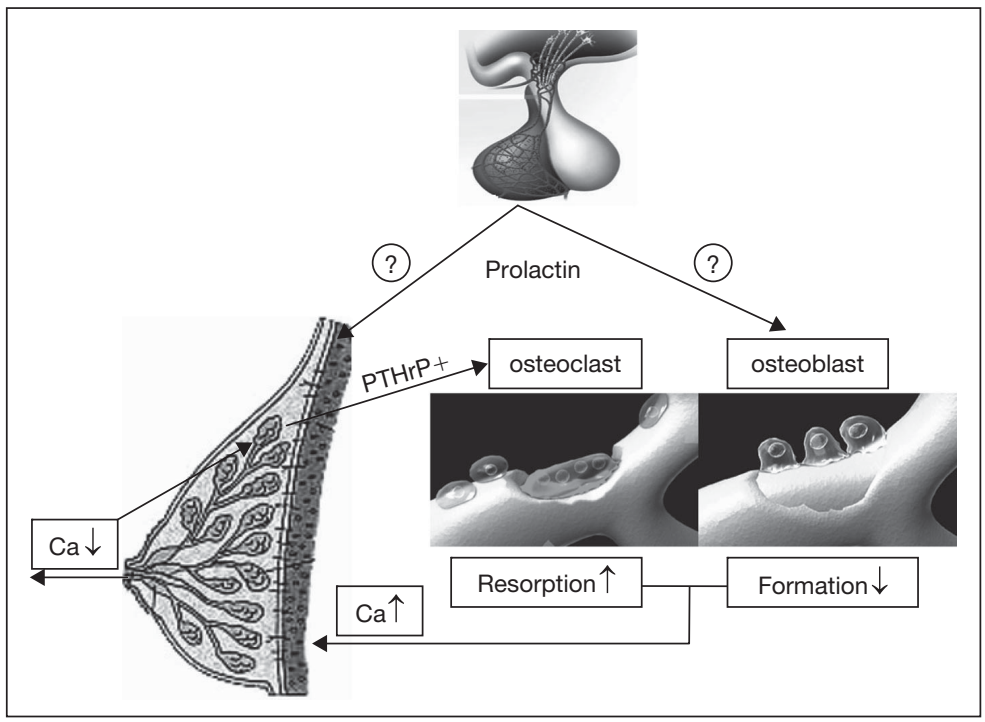

Fig. 1. Hypocalcemia reduces overall production and calcium content in milk. The lactating mammary gland can sense the extracellular calcium through a calcium-sensing receptor and adjust its secretion to the milk and increases PTHrP secretion from the mammary epithelial cells. The combination of low estrogens and high PTHrP during lactation enhances bone resorption. This was one side of the circle, now we learn that prolactin, the most important hormone during lactation, also suppresses osteoblast differentiation and maturation and by that inhibits bone remodeling and calcium deposition in bone. 
comment, decreased circulating levels of estrogen, and a negative calcium balance [6]. When calcium becomes limited, calcium restriction decreases the calcium content of milk, increases milk osmolality and protein concentration, and decreases overall milk production. In order to prevent this cascade, PTHrP is secreted from the mammary gland to act on the maternal bone, the physiological calcium reservoir to enhance calcium release [7]. From this paper, we have now learned that prolactin, the most important hormone for lactation, also plays a physiological role to decrease osteoblast proliferation which leads to a reduction of new bone formation (fig. 1).

\section{Mechanism of the year: now we know how calcium modulates skeletal development}

\section{The extracellular calcium-sensing receptor (CaSR) is a critical modulator of skeletal development}

Chang W, Tu C, Chen TH, Bikle D, Shoback D

Endocrine Research Unit, Department of Veterans Affairs Medical Center, Department of Medicine, University of

California, San Francisco, Calif., USA

wenhan.chang@ucsf.edu

Sci Signal 2008; 1:ra1

Background: The extracellular $\mathrm{Ca}^{2+}$-sensing receptor (CaSR) is well known to play an important role in the functions of the parathyroid gland and the kidney. Severe hyperparathyroidism, premature death, and incomplete gene excision in $\mathrm{Casr}^{-/-}$mice have precluded the assessment of CaSR function in other tissues.

Methods: The authors generated mice with tissue-specific deletion of Casr in the parathyroid gland, bone, or cartilage.

Results: Deletion of Casr in the parathyroid gland or bone was found to result in profound bone defects, whereas deletion of Casr in chondrocytes resulted in death before embryonic day 13 (E13). Mice in which chondrocyte-specific deletion of Casr was induced between E16 and E18 were viable but showed delayed growth plate development.

Conclusion: The data show a critical role for the CaSR in early embryogenesis and skeletal development.

Heterozygous and homozygous inactivating mutations in Casr cause familial benign hypocalciuric hypercalcemia (FBHH) and neonatal severe hyperparathyroidism (NSHPT), respectively [8]. Patients with NSHPT have severe skeletal demineralization at birth, which is thought to be due to severe hyperparathyroidism. The presence of CaSRs in bone and cartilage has, however, raised the question of whether defective signaling of CaSRs in bone cells, chondrocytes, or both may contribute to the skeletal phenotype in NSHPT. The authors unexpectedly observed a marked reduction in the abundance of the CaSR in the bones of mice with tissue-specific Casr deletion in the parathyroid gland, which suggests that loss of CaSR signaling in that tissue may also contribute to skeletal pathology. This study provides the first evidence supporting yet another level of coordination between the parathyroid gland and bone, which is mediated by CaSR, and the direct involvement of CaSR signaling in the differentiation of osteoblasts and growth plate chondrocytes and skeletal development in vivo. Furthermore, it suggests that inhibition of Casr expression in bone in states of hyperparathyroidism may be part of the mechanism underlying the skeletal defects in this disorder. It remains unclear why the expression of CaSR is decreased in bone when Casr is specifically knocked out only in the parathyroid gland. The hypercalcemic phenotype might regulate the abundance of Casr in bone by a feedback loop. 


\section{PTH(1-34) replacement therapy in a child with hypoparathyroidism caused by a sporadic calcium receptor mutation}

Theman TA, Collins MT, Dempster DW, Zhou H, Reynolds JC, Brahim JS, Roschger P, Klaushofer K, Winer KK

Craniofacial and Skeletal Diseases Branch, National Institute of Dental and Craniofacial Research, National Institutes

of Health, Department of Health and Human Services, Bethesda, Md., USA

mc247k@nih.gov

J Bone Miner Res 2009;24:964-973

Background: Autosomal dominant hypocalcemia $(\mathrm{ADH})$ causes hypoparathyroidism due to activating mutations in the calcium-sensing receptor (CaSR). Treatment with parathyroid hormone, $\mathrm{PTH}(1-34)$, may be superior to conventional therapy but is contraindicated in children, and long-term effects on the skeleton are unknown.

Methods: A 20-year-old female with ADH was treated with PTH continuously for 14 years since 6 years and 2 months of age. A bone biopsy was obtained for histomorphometry and quantitative backscattered electron imaging (qBEI). These results were compared with on1e age-, sex-, and length of hypoparathyroidism-matched control not on PTH and 2 sex-matched ADH controls before and after 1 year of PTH.

Results: The patient's growth was normal. Hypercalciuria and hypermagnesuria persisted despite normal or subnormal serum calcium and magnesium levels. Nephrocalcinosis developed by 19 years of age. Cancellous bone volume was dramatically elevated in the patient and in ADH controls after 1 year of PTH. Bone mineral density distribution by qBEI of the patient and ADH controls was strikingly shifted toward lower mineralization compared with the non-ADH control. Moreover, the ADH controls exhibited a further reduction in mineralization after 1 year of PTH. There were no fractures or osteosarcoma.

Conclusions: Long-term PTH replacement in a child with ADH was safe, increased bone mass without negatively impacting mineralization, and improved serum mineral control but did not prevent nephrocalcinosis. These findings imply a role for the CaSR in bone matrix mineralization.

\section{Effects of once versus twice-daily parathyroid hormone 1-34 therapy in children with hypoparathyroidism}

Winer KK, Sinaii N, Peterson D, Sainz B Jr, Cutler GB Jr

National Institute of Child Health and Human Development, National Institutes of Health, Bethesda, Md., USA

winerk@mail.nih.gov

J Clin Endocrinol Metab 2008;93:3389-3395

Background: Long-term conventional therapy in hypoparathyroidism with vitamin D and analogs may lead to nephrocalcinosis and renal insufficiency. The aim of the study was to compare the response of once-daily vs. twice-daily PTH(1-34) treatment in children with hypoparathyroidism.

Methods: Fourteen children ages 4-17 years with chronic hypoparathyroidism were studied. In a randomized cross-over trial, lasting 28 weeks, which compared two dose regimens, once-daily vs. twicedaily PTH(1-34). Each 14-week study arm was divided into a 2-week inpatient dose-adjustment phase and a 12-week outpatient phase.

Results: Repeated serum measures over a 24-hour period showed that twice-daily PTH(1-34) increased serum calcium and magnesium levels more effectively than a once-daily dose. This was especially evident during the second half of the day (12-24 h). PTH(1-34) normalized mean 24-hour urine calcium excretion on both treatment schedules. This was achieved with half the $\mathrm{PTH}(1-34)$ dose during the twice-daily regimen compared with the once-daily regimen (twice-daily $25 \pm 15 \mu \mathrm{g} /$ day vs. once-daily 58 $\pm 28 \mu \mathrm{g} /$ day; $\mathrm{p}<0.001$ ).

Conclusions: The results showed, as in the previous study of adult patients with hypoparathyroidism, that a twice-daily regimen produced significantly improved metabolic control compared with once-daily PTH(1-34). 
Since 2002, recombinant human PTH(1-34) (teriparatide) has been approved for the treatment of postmenopausal osteoporosis. In these 2 papers from the same center it was shown that a twice-daily regimen produced significantly improved metabolic control compared with once-daily PTH(1-34) when given to pediatric patients. As to the long-term effect of recombinant human PTH(1-34) on bone mineral density in patients with a mutated CaSR, it was found that the cancellous bone volume dramatically increased. Based on studies that reported an increased incidence of bone neoplasms in juvenile Fischer 344 rats receiving daily high-dose injections of PTH(1-34) for 2 years (the average lifespan of rats), the US Food and Drug Administration added a 'black-box' warning regarding osteogenic sarcoma in the rat model $[9,10]$. Under this 'black-box' warning it should be emphasized that treatment with recombinant human PTH(1-34) should be restricted only to controlled studies during infancy, childhood or puberty.

\section{Clinical trials: effectiveness of vitamin D supplementation is independent of dosing regimen}

\section{Treatment of hypovitaminosis $D$ in infants and toddlers}

Gordon CM, Williams AL, Feldman HA, May J, Sinclair L, Vasquez A, Cox JE

Divisions of Adolescent Medicine and Endocrinology, Children's Hospital Boston, Boston, Mass., USA

catherine.gordon@childrens.harvard.edu

J Clin Endocrinol Metab 2008;93:2716-2721

Background: Hypovitaminosis D appears to be on the rise in young children, with implications for skeletal and overall health. The objective of the study was to compare the safety and efficacy of vitamin $\mathrm{D}_{2}$ daily, vitamin $\mathrm{D}_{2}$ weekly, and vitamin $\mathrm{D}_{3}$ daily, combined with supplemental calcium, in raising serum 25-hydroxyvitamin D [25(OH)D] and lowering PTH concentrations.

Methods: This was a 6-week randomized controlled trial. Forty otherwise healthy infants and toddlers with hypovitaminosis D $[25(\mathrm{OH}) \mathrm{D}<20 \mathrm{ng} / \mathrm{ml}]$ were enrolled. Participants were assigned to 1 of 3 regimens: 2,000 IU oral vitamin $\mathrm{D}_{2}$ daily, 50,000 IU vitamin $\mathrm{D}_{2}$ weekly, or 2,000 IU vitamin $\mathrm{D}_{3}$ daily. Each was also prescribed elemental calcium $(50 \mathrm{mg} / \mathrm{kg} / \mathrm{day})$. Infants received treatment for 6 weeks.

Results: All treatments approximately tripled the $25(\mathrm{OH}) \mathrm{D}$ concentration. Preplanned comparisons were nonsignificant: daily vitamin $\mathrm{D}_{2}$ vs. weekly vitamin $\mathrm{D}_{2}(12 \%$ difference in effect, $\mathrm{p}=0.66)$ and daily $\mathrm{D}_{2}$ vs. daily $\mathrm{D}_{3}(7 \%, \mathrm{p}=0.82)$. The mean serum calcium change was small and similar in the 3 groups. There was no significant difference in PTH suppression.

Conclusions: The short-term vitamin $\mathrm{D}_{2} 2,000$ IU daily, vitamin $\mathrm{D}_{2}$ 50,000 IU weekly, or vitamin $\mathrm{D}_{3} 2,000$ IU daily yielded equivalent outcomes in the treatment of hypovitaminosis D among young children. Therefore, pediatric providers can individualize the treatment regimen for a given patient to ensure compliance, given that no difference in efficacy or safety was noted among these 3 common treatment regimens.

Modern lifestyle sustains a high incidence of hypovitaminosis $D$, which is the most frequent cause for rickets. Although the recommendations for vitamin D supplementation are well known [11], compliance with vitamin D supplementation is often a problem. The aim of the present study was to examine the efficacy of each treatment in raising serum 25(OH)D and lowering PTH concentrations; and the safety and tolerance of each regimen in infants and toddlers treated with either a daily low dose of vitamin $D_{2}$, a higher dose of vitamin $D_{2}$ once weekly, or a low dose of vitamin $D_{3}$ once daily. Sun exposure and fish consumption provide vitamin $D_{3}$ as cholecalciferol while vitamin $D_{2}$ is a plantderived form of vitamin $D$ produced through ultraviolet exposure of foods (ergocalciferol). Both vitamin $D_{2}$ and vitamin $D_{3}$ when ingested undergo metabolism in the liver to form 25 -hydroxyvitamin $D\left[25(\mathrm{OH}) \mathrm{D}\right.$; $\mathrm{D}$ represents either $\mathrm{D}_{2}$ or $\mathrm{D}_{3}$ ] and in the kidneys to 1,25 -dihydroxyvitamin $\mathrm{D}$ [12]. The question whether vitamin $D_{2}$ is as effective as vitamin $D_{3}$ in maintaining 25 -hydroxyvitamin $D$ status was investigated and reassured in healthy adults [13]. In this study it was demonstrate that 2,000 IU daily vitamin $D_{2}, 50,000$ IU vitamin $D_{2}$ weekly, or 2,000 IU daily vitamin $D_{3}$ yield equivalent outcomes in the short-term treatment of hypovitaminosis $D$ among otherwise healthy infants and toddlers. 
These results indicate that pediatric providers can determine the appropriate method of treatment for a given patient or family to ensure compliance, given that no difference in efficacy or safety was noted.

\title{
Concept revisited: longitudinal assessment of the effects of estrogen receptor- $\alpha$ deletion on human bone
}

\author{
Impact on bone of an estrogen receptor- $\alpha$ gene loss of function mutation \\ Smith EP, Specker B, Bachrach BE, Kimbro KS, Li XJ, Young MF, Fedarko NS, Abuzzahab MJ, Frank GR, Cohen RM, \\ Lubahn DB, Korach KS \\ Division of Endocrinology, Diabetes and Metabolism, Department of Medicine, University of Cincinnati College of \\ Medicine, Vontz Center for Molecular Studies, Cincinnati, Ohio, USA \\ smithep@email.uc.edu \\ J Clin Endocrinol Metab 2008;93:3088-3096
}

Background: There exists only one kindred with a known instance of a germ line loss of function mutation of estrogen receptor- $\alpha(E R \alpha)$. The aim was to assess the impact of a loss of function mutation in the $\mathrm{ER} \alpha$ gene on bone histomorphometry, bone volumetric density, bone geometry and skeletal growth, and to evaluate the effect of ER $\alpha$ heterozygosity on spine density and adult height in an extended pedigree.

Methods: A longitudinal follow-up of the propositus with homozygous loss of function mutation of ER $\alpha$ and a single contact evaluation of the kindred were performed. Iliac crest bone biopsy and peripheral quantitative computed tomography (pQCT) of propositus with serial measures of spine areal bone mineral density (aBMD) by dual-energy X-ray absorptiometry (DXA) and bone age were performed. Members of the pedigree were evaluated for ER $\alpha$ mutation carrier status and spine aBMD.

Results: Bone biopsy revealed marked osteopenia, low trabecular volume, decreased thickness, normal trabecular number, and low activation frequency. Radial periosteal circumference was similar, endosteal circumference larger, and trabecular and cortical volumetric BMD markedly lower than controls. Spine aBMD at age 28.5 years was $0.745 \mathrm{~g} / \mathrm{cm}^{2}$ which decreased to $0.684 \mathrm{~g} / \mathrm{cm}^{2}(Z$ score -3.85$)$ at 35.5 years while bone age advanced from 15.0 to 17.5 years. Kindred analysis revealed that gene carriers had spine aBMD Z scores of $<0(\mathrm{p}=0.003)$, although no difference was observed between carriers and nonmutant members $(-0.84 \pm 0.26$ vs. $-0.64 \pm 0.16)$.

Conclusion: Homozygous ER $\alpha$ disruption markedly affects bone growth, bone mineral content and bone structure but not periosteal circumference. ER $\alpha$ heterozygosity appears to not impair spine aBMD.

Estrogens are known to be important for skeletal maturation. There is only one known human instance of a disruptive mutation in $E R \alpha$, causing estrogen resistance [14]. When the phenotype of this tall 28-year-old 'Cincinnati man' was first reported, he had masculinized normally in adolescence but had never experienced a growth spurt. At the age of 28 years, he was still growing with a height of $208 \mathrm{~cm}$ and bone age of 15 years. He had developed genu valgum and marked axillary acanthosis nigricans. Gonadotropin and estrogen levels were increased and testosterone concentrations were in the adult normal range. In addition, it was shown that bone mineral density as measured by DXA was markedly reduced. In the current paper, Smith et al. have longitudinally assessed the bone phenotype of the propositus and performed ER $\alpha$ mutation carrier analysis of all family members who provided consent. This study provides additional substantial evidence for an important role for ER $\alpha$ in bone metabolism and indicates that there exists a complex contribution of both estrogens and androgens in bone growth, bone mineral content acquisition and structural integrity of the skeleton. A striking finding in his skeleton is marked thinning of the cortex with increased trabecularization. This may shed light on the debate of bone fragility in the estrogen-deficient Turner patients, who have normal $B M D$, but fragile cortical bones. Interestingly, haploinsufficiency for $E R \alpha$ is not associated with osteopenia or tall stature, indicating that only a major disruption in ER $\alpha$ expression has a clear impact on the skeleton. 


\section{The role of the G protein-coupled receptor GPR30 in the effects of estrogen in ovariectomized mice}

Windahl SH, Andersson N, Chagin AS, Martensson UE, Carlsten H, Olde B, Swanson C, Moverare-Skrtic S, Sävendahl L, Lagerquist MK, Leeb-Lundberg LM, Ohlsson C

Institute of Medicine, Sahlgrenska Academy, Göteborg University, Göteborg

Claes.Ohlsson@medic.gu.se

Am J Physiol Endocrinol Metab 2009;296:E490-496

Background: In vitro studies suggest that the membrane G protein-coupled receptor GPR30 is a functional estrogen receptor (ER). The aim of the present study was to determine the possible in vivo role of GPR30 as a functional ER primarily for the regulation of bone mass and longitudinal bone growth, but also for some other well-known estrogen-regulated parameters.

Methods: Three-month-old ovariectomized GPR30-deficient mice $\left(G P R 30^{-1-}\right)$ and wild-type (WT) mice were treated with either vehicle or increasing doses of estradiol $\left(\mathrm{E}_{2} ; 0,30,70,160\right.$, or $830 \mathrm{ng} / \mathrm{mouse} /$ day). Body composition [bone mineral density (BMD), fat mass, and lean mass] was analyzed by dualenergy-X ray absorptiometry, while the cortical and trabecular bone compartments were analyzed by peripheral quantitative computerized tomography. Quantitative histological analyses were performed in the distal femur growth plate. Bone marrow cellularity and distribution were analyzed using a fluorescence-activated cell sorter.

Results: $\mathrm{E}_{2}$ treatment reduced longitudinal bone growth, reflected by decreased femur length and distal femur growth plate height, in the WT mice but not in the GPR $30^{-1-}$ mice compared with vehicle-treated mice. On the other hand, the estrogenic responses on all other investigated parameters, including increase in bone mass (total body BMD, spine BMD, trabecular BMD, and cortical bone thickness), increase in uterine weight, thymic atrophy, fat mass reduction, and increase in bone marrow cellularity, were similar for all of the investigated $\mathrm{E}_{2}$ doses in WT and GPR $30^{-1-}$ mice.

Conclusion: These in vivo findings demonstrate that GPR30 is required for a normal estrogenic response in the growth plate. In contrast, GPR30 is not required for normal estrogenic responses on several major well-known estrogen-regulated parameters.

Estrogens exert a variety of important physiological effects which have been suggested to be mainly mediated via the two known nuclear estrogen receptors (ERs), ER $\alpha$ and ERß. The 'Cincinnati man' with a point mutation in the ER $\alpha$ had nonfused growth plates and continued to grow after sexual maturation, indicating that $E R \alpha$ is required for the effects of estrogens on the human growth plate [14]. In vitro studies [15] have suggested that the membrane G protein-coupled receptor GPR30 is also a functional ER. Using a newly developed $G P R 30^{-1-}$ mouse model, the authors recently demonstrated that GPR30 deletion abolished $\mathrm{E}_{2}$-stimulated insulin release both in vivo and in vitro [16]. However, the in vivo role of GPR30 as a functional ER for the regulation of skeletal parameters, including bone mass and longitudinal bone growth, has so far been unknown. In the present paper, the same authors present data supporting that GPR30 is required for a normal estrogenic response in the growth plate suggesting that GPR30 may work in concert with the nuclear ERs in mediating estrogenic effects in growth plate cartilage. However, it should be noted that vehicle-treated GPR30${ }^{1-}$ mice displayed reduced growth plate height compared with vehicle-treated WT mice and that this was associated with a nonsignificant trend of reduced femur length in the vehicle-treated $G P R 30^{-1-}$. Thus, one cannot exclude that the lack of a significant $\mathrm{E}_{2}$ effect in the growth plates of $G P R 30^{-1-}$ mice is a consequence of reduced growth plate activity already existing in vehicle-treated $G P R 30^{-1-}$ mice. Nevertheless, a role of GPR30 in the growth plate is supported by recent findings [3] that GPR30 immunoreactivity is present in the human growth plate with the highest expression in the hypertrophic zone. In addition, GPR30 immunoreactivity was demonstrated to decline during pubertal progression, suggesting that GPR30 might be involved in the modulation of longitudinal bone growth [17]. It can be concluded that although GPR30 seems not to be a functional ER in several major ER-responsive tissues, it is mediating $\mathrm{E}_{2}$-stimulated insulin release and is required for a normal estrogenic response in the growth plate. GPR30 might be a future therapeutic target for the modulation of longitudinal bone growth. 


\section{Deleterious effect of late menarche on distal tibia microstructure in healthy 20-year-old and premenopausal middle-aged women}

Chevalley T, Bonjour JP, Ferrari S, Rizzoli R

Division of Bone Diseases, Department of Rehabilitation and Geriatrics, Geneva University Hospitals and Faculty of

Medicine, Geneva, Switzerland

Thierry.Chevalley@hcuge.ch

J Bone Miner Res 2009;24:144-152

Background: Late menarche is a known risk factor for fragility fractures. The authors hypothesized that pubertal timing-dependent alterations in bone structural components would persist from peak bone mass to menopause, independent of premenopausal bone loss.

Methods: The influence of menarcheal age on femoral neck aBMD by DXA and microstructure of distal tibia by high resolution pQCT were studied in healthy young adult (age $20.4 \pm 0.6$ [SD] years, $\mathrm{n}=124$ ) and premenopausal middle-aged (age $45.8 \pm 3.4$ years, $n=120$ ) women. Median menarcheal age was $13.0 \pm 1.2$ and $13.1 \pm 1.7$ years in young adult and premenopausal middle-aged women, respectively. Results: In young adults and premenopausal middle-aged women $(\mathrm{n}=244)$, femoral neck aBMD, total volumetric BMD and cortical thickness of distal tibia were inversely correlated to menarcheal age. After segregation by the median menarcheal age in early and late subgroups, the significant influences of both menarcheal age $(\mathrm{p}=0.004)$ and chronological age $(\mathrm{p}<0.0001)$ were observed for femoral neck aBMD and trabecular bone volume fraction of the distal tibia with similar differences in $\mathrm{T}$ scores between late and early subgroups in young adults and premenopausal middle-aged women. Cortical thickness was negatively influenced by menarcheal age, whereas trabecular thickness was negatively influenced by chronological age. There was also a striking inverse relationship between cross-sectional area and cortical thickness $(\mathrm{R}=-0.57, \mathrm{p}<0.001)$.

Conclusion: The negative influence of late menarcheal age at weight-bearing sites remained unattenuated a few years before menopause and was independent of premenopausal bone loss. Alterations in both bone mineral mass and microstructural components may explain the increased risk of fragility fractures associated with later menarcheal age.

Several factors in women that are related to their reproductive life influence bone health. The age at menarche and variations in pubertal timing are known to influence the risk of fragility fractures [18]. In the current paper, Chevalley et al. aimed to study whether the influence of menarcheal age at the time of peak bone mass attainment was also detectable in a cohort of premenopausal women close to the mean age of menopause onset. The study design allowed testing the hypothesis of whether any deleterious effect of premenopausal bone loss in healthy women would be additive to the persisting negative influence of late menarche. Based on their findings, it appears that pubertal timing in women is an important factor modulating the risk of sustaining a fragility fracture during adulthood. The deficit observed at the age of peak bone mass, which is attributable to late pubertal timing, remained evident and significant even 25 years later at an age close to the onset of menopause. In healthy women, a delay of approximately 2 years in menarcheal age was linked to significant decreases in T scores of the femoral neck aBMD and trabecular density at the distal tibia. The launch of the World Health Organization technical report 'Assessment of osteoporosis at the primary health care level' and the related web-based FRAX ${ }^{\circledR}$ tool (http://www.shef.ac.uk/FRAX/) have been major milestones towards helping health professionals worldwide to improve identification of patients at high risk of fracture. An individual's risk factors such as age, sex, weight, height, and femoral neck BMD (if available) are entered into the website tool, followed by clinical risk factors which include a prior fragility fracture, parental history of hip fracture, current tobacco smoking, long-term use of glucocorticoids, rheumatoid arthritis, other causes of secondary osteoporosis and daily alcohol consumption. Before late menarcheal age can be included as a risk factor for females in the FRAX ${ }^{\circledast}$ tool, the data in this paper must be confirmed in a larger population and the background conditions leading to late menarche need to be better scrutinized. 


\title{
Sclerostin antibody treatment increases bone formation, bone mass, and bone strength in a rat model of postmenopausal osteoporosis
}

\author{
Li X, Ominsky MS, Warmington KS, Morony S, Gong J, Cao J, Gao Y, Shalhoub V, Tipton B, Haldankar R, Chen Q, \\ Winters A, Boone T, Geng Z, Niu QT, Ke HZ, Kostenuik PJ, Simonet WS, Lacey DL, Paszty C \\ Department of Metabolic Disorders, Amgen, Thousand Oaks, Calif., USA \\ cpatzty@amgen.com \\ J Bone Miner Res 2009;24:578-588
}

Background: A long-standing goal in the treatment of bone loss conditions, such as osteoporosis, has been the development of bone-rebuilding anabolic agents. The osteocyte-derived secreted protein sclerostin has been shown to be a key negative regulator of bone formation, although the magnitude and extent of sclerostin's role in the aging skeleton is still unclear. The aim was to study sclerostin biology and to assess the pharmacologic effects of sclerostin inhibition.

Methods: The authors used a cell culture model of bone formation to identify a promising sclerostinneutralizing monoclonal antibody (Scl-AbII), which then was tested in an aged ovariectomized rat model of postmenopausal osteoporosis. Six-month-old female rats were ovariectomized and left untreated for 1 year to allow significant estrogen deficiency-induced bone loss, at which point Scl-AbII was administered for 5 weeks.

Results: Scl-AbII treatment in these animals had robust anabolic effects, with marked increases in bone formation on trabecular, periosteal, endocortical, and intracortical surfaces. This resulted in complete reversal of estrogen deficiency-induced bone loss at several skeletal sites, but also further increased bone mass and bone strength to levels greater than those found in non-ovariectomized control rats.

Conclusion: These preclinical results establish sclerostin's role as a pivotal negative regulator of bone formation in the aging skeleton. In addition, antibody-mediated inhibition of sclerostin represents a promising new therapeutic approach for the anabolic treatment of bone-related disorders.

The vast majority of therapeutics that are being used in the treatment of bone loss conditions are antiresorptive agents (e.g. bisphosphonates) that exert their clinical effect by decreasing the rate of bone loss and achieving a reduction in fracture incidence. However, there has been a long-standing goal to develop bone-rebuilding anabolics. Currently, the only approved bone anabolic is parathyroid hormone which, either in full-length or truncated form, is administered by daily injections. A new possible target for bone anabolic treatment has emerged from the discovery of a protein called sclerostin. The sclerostin gene was originally identified by mutational analyses of an extremely rare genetic disorder, sclerosteosis (Van Buchem's disease), which is an autosomal recessive sclerosing bone dysplasia characterized by massive bone overgrowth [19]. Sclerostin has been demonstrated to block bone formation both in vivo and in vitro. It is produced by mature osteocytes and has not been found in any other cell type, making it very bone-specific and thus an optimal drug candidate. The authors of this elegant article developed, screened and tested a particular sclerostin antibody, which resulted in a complete reversal of estrogen deficiency-induced bone loss. More dramatically, bone mass and strength were further increased to greater levels than in controls. These results indicate that osteocyte-derived sclerostin is a pivotal negative regulator of bone formation, bone mass and bone strength, and has great clinical potential. The osteocyte has long been hypothesized to orchestrate local bone remodeling and the value of this cell type as a drug discovery target is becoming more evident. As reviewed in Yearbook 2008 [3], osteocytes can control physiological functions distant from bone by the production of osteocalcin and FGF-23 but clearly also have local effects on skeletal metabolism via sclerostin. Interestingly, sclerostin was recently also found to play an essential role in mediating bone response to mechanical unloading, an effect which was found to be mediated through Wnt/ $\beta$-catenin signaling [20]. Wnt/ $\beta$-catenin signaling plays a key role in controlling bone mass via regulating multiple aspects including osteoblast differentiation and function and its activity has also previously been shown to be enhanced upon mechanical loading [21]. 


\section{Vitamin D receptor genotype in hypophosphatemic rickets as a predictor of growth and response to treatment}

Jehan F, Gaucher C, Nguyen TM, Walrant-Debray O, Lahlou N, Sinding C, Dechaux M, Garabedian M Institut National de la Santé et de la Recherche Médicale Unit 561, Hôpital Saint Vincent de Paul, Paris, France frederic.jehan@inserm.fr

J Clin Endocrinol Metab 2008;93:4672-4682

Background: Treatment of X-linked hypophosphatemic rickets improves bone mineralization and bone deformities, but its effect on skeletal growth is highly variable. The aim of the study was to explore whether genetic variants in the promoter region of the vitamin D receptor (VDR) gene may explain the response to treatment.

Methods: The VDR promoter haplotype structure was studied in a large cohort of 91 patients with hypophosphatemic rickets including 62 patients receiving $1 \alpha$-hydroxyvitamin $\mathrm{D}_{3}$ derivatives and phosphates from early childhood.

Results: Treatment improved bone deformities and final height, but 39\% of treated patients still had short stature at the end of growth ( -2 SD score or below). Height was closely associated with VDR promoter Hap1 genotype. Hap1- patients $(35 \%$ of the cohort) had severe growth defects. This disadvantageous association of Hap1- status with height was visible before treatment, under treatment, and on to adulthood. Compared with Hap1 ${ }^{+}$patients, those who were Hap1- had a higher urinary calcium response to $1 \alpha$-hydroxyvitamin $\mathrm{D}_{3}$ and had significantly lower circulating FGF23 levels.

Conclusions: The VDR promoter genotype can serve as a key predictor of growth under treatment with $1 \alpha$-hydroxyvitamin $D_{3}$ derivatives in patients with hypophosphatemic rickets, including those with established PHEX alterations. The VDR promoter genotype appears to provide valuable information for adjusting treatment and for deciding upon the utility of early GH therapy.

Children with hypophosphatemic rickets grow poorly and have a shorter than expected final height. In this study the authors found that analysis of VDR promoter genotype appears to be a valuable tool for predicting the severity of the growth defect and the risk of developing hypercalciuria. The severe growth defect in Hap1- ${ }^{-}$patients cannot easily be attributed to a higher sensitivity to vitamin D and was obviously disconnected from the severity of bone deformities and from the biological phenotype. The present data are at odds with that obtained in healthy adolescents, showing that girls with a Hap1- genotype were taller, not shorter, than girls with a Hap1+ genotype [22]. This suggests a currently unknown local VDR effect on cell proliferation or differentiation in the growth plates of long bones that involves one or more factors altered by mutations of the Phex gene. Further studies are needed to conclude whether VDR promoter genotype analysis will play a role in defining an optimal therapeutic strategy that includes GH treatment in hypophosphatemic patients. Genotype-phenotype inconsistencies were puzzling when we used to think of monogenic diseases. In the new era of genome-wide association studies nothing remains monogenic, and VDR polymorphism (certainly not the only one) may contribute to the phenotype variability in Phex gene mutations.

\section{New concerns: new anti-cancer and anti-inflammatory drugs may interfere with bone growth}

\section{Damaging effects of chronic low-dose methotrexate usage on primary bone formation in young rats and potential protective effects of folinic acid supplementary treatment}

Fan C, Cool JC, Scherer MA, Foster BK, Shandala T, Tapp H, Xian CJ

Department of Orthopaedic Surgery, Women's and Children's Hospital, Adelaide, SA, Australia

cory.xian@unisa.edu.au

Bone 2009;44:61-70

Background: Methotrexate (MTX) is an often used anti-metabolite in cancer treatment and is frequently used as an anti-rheumatic drug. While MTX chemotherapy at a high dose is known to cause bone 
growth defects in growing bones, the effects of its chronic use at a low dose on the growing skeleton remain less clear.

Methods: The authors examined the effects on bone growth of long-term MTX chemotherapy at a low dose in young rats, and potential protective effects of supplementary treatment with the antidote folinic acid (given i.p. at $1 \mathrm{mg} / \mathrm{kg} 6 \mathrm{~h}$ after MTX).

Results: Two cycles of 5 once-daily MTX injections $(0.75 \mathrm{mg} / \mathrm{kg}, 5$ days on $/ 9$ days off $/ 5$ days on $)$ caused a significant reduction in the heights of growth plate and primary spongiosa bone when analyzed on day 22 and compared to controls. In contrast, a similar dosing regimen but at a lower dose $(0.4 \mathrm{mg} / \mathrm{kg})$ caused only slight or no reduction in heights of both regions. However, after the induction phase at this $0.4 \mathrm{mg} / \mathrm{kg}$ dosing, continued use of MTX at a low dose (once weekly at $0.2 \mathrm{mg} / \mathrm{kg}$ ) caused a reduction in primary spongiosa height and bone volume in weeks 9 and 14, which was associated with an increased osteoclast formation and decreased osteoblast bone surface density in the primary spongiosa. Folinic acid supplementation prevented the MTX effects in the primary spongiosa.

Conclusion: The data show that acute use of MTX can damage growth plate and primary bone at a high dose, but not at a low dose. However, long-term use of MTX at a low dose can reduce primary bone formation, an effect which is prevented by folic acid supplementation.

The intensive use of chemotherapy with anti-cancer drugs has been commonly associated with bone growth arrest and osteoporosis in survivors, and it has also become apparent that children are showing poor bone growth after chemotherapy has stopped. Methotrexate (MTX), an often used anticancer and anti-rheumatic drug, acts as an anti-folate metabolite affecting purine/thymidylate and thus DNA synthesis and cell proliferation. Despite the effectiveness of MTX in both cancer chemotherapy and rheumatoid arthritis management, discontinuation is common due to the occurrence of its adverse side effects. MTX is known to cause bone growth defects in pediatric cancer patients, and fractures in children have been reported during and after chemotherapy treatment [23], which may be due to the accumulation of dose during long-term chemotherapy treatment. The authors hypothesized that the damaging effects of MTX on bone growth and bone formation will be treatment dosage- and duration-dependent and that supplementary folinic acid may provide protective effects during long-term low-dose MTX chemotherapy. Their data show that acute use of MTX can damage growth plate and primary bone at a high dose, but not at a low dose. However, long-term use of MTX at a low dose also reduced primary bone formation, an effect which was prevented by folic acid supplementation. Further studies are needed to characterize the MTX skeletal damaging effects and the underlying mechanisms and to investigate benefits and possible mechanisms of folinic acid action in protecting bone growth during MTX chemotherapy.

\section{Bone growth during rapamycin therapy in young rats}

Sanchez CP, He YZ

Department of Pediatrics, University of Wisconsin School of Medicine and Public Health, Madison, Wisc., USA

cpsanchez@pediatrics.wisc.edu

BMC Pediatr 2009;9:3

Background: Rapamycin is an effective immunosuppressant widely used to maintain the renal allograft in pediatric patients. As rapamycin has potent anti-proliferative and anti-angiogenic properties, it was hypothesized that linear growth may be adversely affected if used in growing individuals.

Methods: Weanling 3-week-old rats were given rapamycin $(2.5 \mathrm{mg} / \mathrm{kg})$ daily by gavage for 2 or 4 weeks. Serum levels of calcium, phosphate, PTH, urea nitrogen, creatinine and insulin-growth factor I (IGF-I) were analyzed. Histomorphometric analysis of the growth plate cartilage, in situ hybridization experiments and immunohistochemical studies for various proteins were performed.

Results: At the end of the 2 weeks, body and tibia length measurements were shorter after rapamycin therapy associated with an enlargement of the hypertrophic zone and a decrease in chondrocyte proliferation in the growth plate cartilage. The increased number of hypertrophic chondrocytes may be partly explained by reduced expression of parathyroid hormone/parathyroid hormone-related peptide (PTH/PTHrP) and an increase in Indian hedgehog (Ihh). In addition, the number of chondro/osteoclasts declined in the chondro-osseous junction. Although body and tibial length remained short after 4 weeks of rapamycin, changes in the expression of chondrocyte proliferation, chondrocyte differentiation and chondro/osteoclastic resorption, which were significant after 2 weeks of rapamycin, improved at the end of 4 weeks. 
Conclusion: When given to young rats, 2 weeks of rapamycin significantly decreased endochondral bone growth. At the end of 4 weeks, no catch-up growth was demonstrated although markers of chondrocyte proliferation and differentiation improved.

Rapamycin is a powerful immunosuppressant widely used in children to maintain the renal allograft. Studies have shown that rapamycin decreases cell proliferation by inhibition of the mammalian target of rapamycin (mTOR), a key regulator in cell growth [24]. In addition, rapamycin has been demonstrated to exert anti-angiogenic properties to control tumor growth by reduction in vascular endothelial growth factor (VEGF) expression [25]. Since rapamycin is now a standard immunosuppressant used to maintain an organ transplant in children, linear growth may be affected if rapamycin is administered long-term to young and growing patients. The authors aimed to assess the short- and long-term effects of rapamycin on endochondral bone growth in young rats with normal renal function using markers of chondrocyte proliferation, chondrocyte differentiation, chondroclast/osteoclastic resorption and angiogenesis in the tibial growth plate. Their data suggest that rapamycin suppresses bone growth, an effect which may be linked to an observed decline in chondrocyte proliferation, enhancement of chondrocyte maturation, and alterations in cartilage resorption and vascularization. The present study showed a downregulation by rapamycin of PTHIPTHrP accompanied by enhancement of $I h h$, a mechanism that has been reviewed in the Yearbook series several times over. Interestingly, the authors found that the 2-week effects of rapamycin on chondrocyte proliferation, chondrocyte maturation and vascular invasion may improve to near normal if rapamycin is administered continuously as the animal matures although no catch-up growth was demonstrated. Therefore, it is too early to draw any conclusions regarding any long-term effects of rapamycin on longitudinal bone growth. Furthermore, it is important to point out that the rapamycin dose used in this study was higher than the currently prescribed amount in pediatric patients. Clinical studies are needed to assess whether long-term therapy with rapamycin can affect linear growth in young pediatric patients.

Food for thought: could aspirin prevent osteoporosis?

\section{Pharmacologic stem cell based intervention as a new approach to osteoporosis treatment in rodents}

Yamaza T, Miura Y, Bi Y, Liu Y, Akiyama K, Sonoyama W, Patel V, Gutkind S, Young M, Gronthos S, Le A, Wang CY, Chen W, Shis

Center for Craniofacial Molecular Biology, University of Southern California School of Dentistry, Los Angeles, Calif., USA

wchen@mail.nih.gov or songtaos@usc.edu

PLOS ONE 2008;3:e2615

Background: Osteoporosis is the most prevalent skeletal disorder, characterized by a low bone mineral density (BMD) and bone structural deterioration, leading to bone fragility fractures. Accelerated bone resorption by osteoclasts has been established as a principal mechanism in osteoporosis. However, recent experimental evidence suggests that apoptosis of osteoblasts and osteocytes would also account for the imbalance in bone remodeling in osteoporosis. The aim was to examine whether aspirin, which has been reported as an effective drug improving bone mineral density in human epidemiology studies, regulates the balance between bone resorption and bone formation at the level of stem cell differentiation.

Methods: The authors performed multiple in vitro and in vivo experiments on activated $\mathrm{T}$ cells and bone marrow mesenchymal stem cells (MSCs). The role of aspirin was evaluated both in vitro and in vivo. The ovariectomy (OVX)-induced osteoporosis model in the mouse was selected to examine the feasibility and mechanisms of aspirin-mediated therapy for osteoporosis.

Results: T-cell-mediated MSC impairment was found to play a crucial role in OVX-induced osteoporosis. Ex vivo mechanistic studies revealed that T-cell-mediated MSC impairment was mainly attributed to the apoptosis of MSCs via the Fas/Fas ligand pathway. Aspirin inhibited T-cell activation and Fas ligand 
induced MSC apoptosis in vitro. Furthermore, aspirin increased osteogenesis of MSCs and inhibited osteoclast activity in OVX mice, leading to ameliorating bone density.

Conclusion: A novel mechanism for osteoporosis in which activated T cells induce MSC apoptosis via the Fas/Fas ligand pathway was identified. The results suggest that pharmacologic stem cell-based intervention by aspirin to activate osteoblasts and inhibit osteoclasts may be a new alternative in the treatment of osteoporosis.

Have you ever thought of aspirin as a potential medicine to restore bone mineral balance after estrogen-deprivation? This complex study comes from 5 different research centers in the US, Japan and Australia, and states two provocative messages. The first is an extension of prior studies on the role of T cells in the pathogenesis of osteoporosis. Previously, the role of lymphocytes in pathological bone loss has focused on the effects on bone resorption. Here, the authors present an impressive number of in vitro and in vivo experiments demonstrating that activated $T$ cells are also involved in the apoptosis of bone marrow MSCs, suggesting that activated T cells may decrease bone formation. The second message is a new finding that pretreatment with aspirin diminishes bone loss in OVX mice by a T-cell-dependent mechanism. OVX mice were pretreated for 2 months with aspirin before surgery and continued on aspirin for another month after OVX. These aspirin-treated OVX mice were compared with sham-operated and OVX mice without any aspirin treatment. Mice treated with aspirin had less trabecular bone loss compared to OVX only mice. However, since there were no shamoperated controls treated with aspirin, it is not clear whether the effects observed with aspirin treatment are specific to OVX. This work certainly gives some food for thought: it suggests that aspirin has a potential to be used as an anti-osteoporosis drug, although many questions still remain. For example, the direct mechanism of aspirin action was not identified in this study. However, since human epidemiologic studies have suggested an association of aspirin with BMD, and since low dose aspirin is widely used and relatively safe, prospective studies of its effects on the rapid peri- and postmenopausal bone loss would be feasible and of great clinical interest.

\title{
Review: bisphosphonates are beneficial in the treatment of pediatric osteogenesis imperfecta patients but long-term effects remain to be clarified
}

\section{Effects of bisphosphonates in children with osteogenesis imperfecta: an AACPDM systematic review}

\author{
Castillo $\mathrm{H}$, Samson-Fang $\mathrm{L}$ \\ Cincinnati Children's Hospital Medical Center, Cincinnati, Ohio, USA \\ Lisa.Samson-Fang@hsc.utah.edu
}

Dev Med Child Neurol 2009;51:17-29

Background: The American Academy for Cerebral Palsy and Developmental Medicine (AACPDM) has undertaken the development of systematic reviews to summarize the literature about specific interventions to assist children with developmental disabilities.

Methods: This systematic review of the effects of bisphosphonate treatment in children with osteogenesis imperfecta $(\mathrm{OI})$ was conducted using methodology for developing systematic reviews of treatment interventions (Revision 1.1) 2004. The review was limited to studies in which bisphosphonate was the intervention and the participants were children with a defined OI. Literature search for studies published in PubMed, CINAHL, and the Cochrane Database of Systematic Reviews was performed. Of 109 citations, 70 met inclusion criteria. Outcomes were classified based on a level of evidence ranking (http://www.cebm.net/index.aspx?o=1047).

Results: Even though a large number of publications on this topic exist, only 8 studies had a sufficiently high level of internal validity to be truly informative. These studies confirmed the improvement in bone density. Many, but not all, studies demonstrated a reduction in fracture rate and enhanced growth. Only very limited evaluation of broader treatment impacts (e.g. deformity, need for orthopedic surgery, pain, functioning, or quality of life) was available. Short-term side effects were reported as minimal. 
Conclusion: The authors conclude that additional research is needed to verify the safe treatment of infants with bisphosphonates. It is not yet clear, which medication and dosing regimen is optimal and how long patients should be treated. The evidence would be strengthened by a larger controlled trial, because many studies lacked adequate power to evaluate stated outcomes. The effects of bisphosphonates in children with milder forms of OI and severe forms that are not due to mutations in the type I pro-collagen gene (e.g. types VII and VIII) are not addressed in the current literature. More studies evaluating especially medication choices, optimal dosing, duration of treatment, post-treatment impacts, and longterm side effects are necessary.

Ol is an inherited bone disease with no cure. Gene therapy has been suggested as one possibility but the variety of mutations and the difficulties in controlling gene expression makes this very distant. Current treatment strategy is based on the use of bisphosphonates to prevent fractures and on surgery. This is a systematic review of the current literature, in which intervention studies with any bisphosphonate on Ol children (aged $<18 \mathrm{yr}$ at time of treatment) were included. Studies were assigned a level of evidence ranking according to study design and methods. Then, the authors coded each level of evidence outcome by a component of the International Classification of Functioning, Disability and Health. Analysis of the component, where the intervention was expected to work (i.e. body function and structure), showed a consistent finding of improved bone density. Reduction in the fracture rate by $30-60 \%$ was demonstrated in 3 of 4 small randomized trials. Statistically significant positive effects on growth were reported in 2 of 5 studies. The authors furthermore evaluated the evidence on the bisphosphonate intervention on other components (e.g. activity, participation) and on the magnitude and nature of medical complications. An encouraging finding was that very few serious shortterm side effects were reported in the published literature and that these side effects were generally mild and reversible. Taken together, this review could confirm the improvement in bone density by bisphosphonate intervention in pediatric Ol patients. A reduction in fracture rate and enhanced growth were demonstrated in many but not all studies. However, little information was available on how long to treat and what the long-term effects are. To conclude, larger controlled trials are needed, since many studies have so far lacked adequate power to evaluate the stated outcomes. Since the use of bisphosphonates is widespread and with the known benefits it is not ethical to perform a randomized controlled trial with an untreated control group. Ideally, future studies should be performed in homogenous groups and should include information on potential confounders. Perhaps a large multicenter study with patients assigned to different dosing regimens could be considered? It is important to point out that while proven to be effective in OI, bisphosphonate therapy in other pediatric patients remains controversial because of inadequate long-term efficacy and safety data. For this reason, many experts recommend limiting the use of these agents to those children with recurrent extremity fractures, symptomatic vertebral collapse, and reduced bone mass. As recently reviewed [26], current data are inadequate to support the use of bisphosphonates in children to treat reductions in bone mass/ density alone. More research is needed to define appropriate indications for bisphosphonate therapy and the optimal agent, dose, and duration of use in pediatric patients.

\section{Review: diagnosing rickets based on the etiology of hypophosphatemia}

\section{Hypophosphatemia: the common denominator of all rickets}

Tiosano D, Hochberg Z

Meyer Children's Hospital, Rambam Medical Center, Haifa, Israel

d_tiosano@rambam.health.gov.il

J Bone Miner Metab 2009; DOI 10.1007/s00774-009-0079-1

Background: Rickets is a disease of hypertrophic growth plate chondrocytes which is caused by hypophosphatemia that leads to a defect in terminal hypertrophic chondrocyte apoptosis. This highlights the critical role of phosphorous in cartilage and bone metabolism.

Results: This paper gives a broad overview of phosphorous metabolism, transport and function in maintaining phosphorous supply to the growth plate, bone osteoblast and the kidney. 
Conclusion: A new classification for the differential diagnosis of rickets is proposed. This is based on the mechanisms leading to hypophosphatemia - high PTH activity, high FGF23 activity or renal phosphaturia.

The common denominator of all rickets is hypophosphatemia. Hypophosphatemia prevents apoptosis in the hypertrophic cells in the growth plate. In the absence of apoptosis, the hypertrophic cells accumulate in the growth plate and form the rachitic bone. Based on these facts, the authors propose that the diagnosis of rickets should be based on the etiologies of hypophosphatemia. The three major entities that can lead to hypophosphatemia are high PTH activity, high FGF23 activity and renal defects that lead to Pi wasting. Hallmarks of high PTH activity are: hypophosphatemia, phosphaturia, disturbance in vitamin D metabolism and low calcium. Hallmarks for high FGF23 activity are hypophosphatemia and phosphaturia with inappropriately low $1,25(\mathrm{OH})_{2} \mathrm{D}$. Hallmarks for renal rickets are hypophosphatemia and phosphaturia with high $1,25(\mathrm{OH})_{2} \mathrm{D}$ that causes hypercalciuria. Besides proposing a new classification of rickets, the review gives an overview of the underlying causes of rickets.

References

1. Rousseau F, Bonaventure J, Legeai-Mallet L, Pelet A, Rozet JM, Maroteaux P, et al: Mutations in the gene encoding fibroblast growth factor receptor-3 in achondroplasia. Nature 1994;371:252-254.

2. Seino Y, Yamanaka Y, Shinohara M, Ikegami S, Koike M, Miyazawa M, et al: Growth hormone therapy in achondroplasia. Horm Res 2000;53(suppl 3):53-56.

3. Chrysis D, Heino T, Sävendahl L: Growth plate, bone and calcium; in Carel J-C, Hochberg Z (eds): Yearbook of Pediatric Endocrinology 2008. Basel, Karger; 2008. pp 63-79.

4. Yasoda A, Komatsu Y, Chusho H, Miyazawa T, Ozasa A, Miura M, et al: Overexpression of CNP in chondrocytes rescues achondroplasia through a MAPK-dependent pathway. Nat Med 2004;10:80-86.

5. Chusho H, Tamura N, Ogawa Y, Yasoda A, Suda M, Miyazawa T, et al: Dwarfism and early death in mice lacking C-type natriuretic peptide. Proc Natl Acad Sci USA 2001;98:4016-4021.

6. VanHouten J, Dann P, McGeoch G, Brown EM, Krapcho K, Neville M, et al: The calcium-sensing receptor regulates mammary gland parathyroid hormone-related protein production and calcium transport. J Clin Invest 2004;113:598608.

7. VanHouten JN, Dann P, Stewart AF, Watson CJ, Pollak M, Karaplis AC, et al: Mammary-specific deletion of parathyroid hormone-related protein preserves bone mass during lactation. J Clin Invest 2003;112:1429-1436.

8. Brown EM: The calcium-sensing receptor: physiology, pathophysiology and CaR-based therapeutics. Subcell Biochem 2007;45:139-167.

9. Vahle JL, Sato M, Long GG, Young JK, Francis PC, Engelhardt JA, et al: Skeletal changes in rats given daily subcutaneous injections of recombinant human parathyroid hormone (1-34) for 2 years and relevance to human safety. Toxicol Pathol 2002;30:312-321.

10. Vahle JL, Long GG, Sandusky G, Westmore M, Ma YL, Sato M: Bone neoplasms in F344 rats given teriparatide $[\mathrm{rhPTH}(1-34)]$ are dependent on duration of treatment and dose. Toxicol Pathol 2004;32:426-438.

11. Wagner CL, Greer FR: Prevention of rickets and vitamin D deficiency in infants, children, and adolescents. Pediatrics 2008;122:1142-1152.

12. Holick MF: Resurrection of vitamin D deficiency and rickets. J Clin Invest 2006;116:2062-2072.

13. Holick MF, Biancuzzo RM, Chen TC, Klein EK, Young A, Bibuld D, et al: Vitamin D2 is as effective as vitamin D3 in maintaining circulating concentrations of 25-hydroxyvitamin D. J Clin Endocrinol Metab 2008;93:677-681.

14. Smith EP, Boyd J, Frank GR, Takahashi H, Cohen RM, Specker B, et al: Estrogen resistance caused by a mutation in the estrogen-receptor gene in a man. N Engl J Med 1994;331:1056-1061.

15. Revankar CM, Cimino DF, Sklar LA, Arterburn JB, Prossnitz ER: A transmembrane intracellular estrogen receptor mediates rapid cell signaling. Science 2005;307:1625-1630.

16. Martensson UE, Salehi SA, Windahl S, Gomez MF, Sward K, Daszkiewicz-Nilsson J, et al: Deletion of the G proteincoupled receptor 30 impairs glucose tolerance, reduces bone growth, increases blood pressure, and eliminates estradiolstimulated insulin release in female mice. Endocrinology 2009;150:687-698.

17. Chagin AS, Savendahl L: GPR30 estrogen receptor expression in the growth plate declines as puberty progresses. J Clin Endocrinol Metab 2007;92:4873-4877.

18. Roy DK, O’Neill TW, Finn JD, Lunt M, Silman AJ, Felsenberg D, et al: Determinants of incident vertebral fracture in men and women: results from the European Prospective Osteoporosis Study (EPOS). Osteoporos Int 2003;14:19-26.

19. Balemans W, Ebeling M, Patel N, Van Hul E, Olson P, Dioszegi M, et al: Increased bone density in sclerosteosis is due to the deficiency of a novel secreted protein (SOST). Hum Mol Genet 2001;10:537-543.

20. Lin C, Jiang X, Dai Z, Guo X, Weng T, Wang J, et al: Sclerostin mediates bone response to mechanical unloading via antagonizing Wnt/beta-catenin signaling. J Bone Miner Res 2009; DOI: 10.1359/JBMR.090411.

21. Robinson JA, Chatterjee-Kishore M, Yaworsky PJ, Cullen DM, Zhao W, Li C, et al: Wnt/beta-catenin signaling is a normal physiological response to mechanical loading in bone. J Biol Chem 2006;281:31720-31728.

22. d'Alesio A, Garabedian M, Sabatier JP, Guaydier-Souquieres G, Marcelli C, Lemacon A, et al: Two single-nucleotide polymorphisms in the human vitamin D receptor promoter change protein-DNA complex formation and are associated with height and vitamin D status in adolescent girls. Hum Mol Genet 2005;14:3539-3548.

23. Mandel K, Atkinson S, Barr RD, Pencharz P: Skeletal morbidity in childhood acute lymphoblastic leukemia. J Clin Oncol 2004;22:1215-1221.

24. Fingar DC, Blenis J: Target of rapamycin (TOR): an integrator of nutrient and growth factor signals and coordinator of cell growth and cell cycle progression. Oncogene 2004;23:3151-3171. 
25. Manegold PC, Paringer C, Kulka U, Krimmel K, Eichhorn ME, Wilkowski R, et al: Antiangiogenic therapy with mammalian target of rapamycin inhibitor RAD001 (Everolimus) increases radiosensitivity in solid cancer. Clin Cancer Res 2008;14:892-900.

26. Bachrach LK, Ward LM: Clinical review 1: Bisphosphonate use in childhood osteoporosis. J Clin Endocrinol Metab 2009;94:400-409. 